\title{
The Treatment of Brain AVMs Study (TOBAS): an all-inclusive framework to integrate clinical care and research
}

\author{
Elsa Magro, MD, ${ }^{1}$ Jean-Christophe Gentric, MD, PhD, ${ }^{2}$ André Lima Batista, MD, ${ }^{3}$ \\ Marc Kotowski, MD, ${ }^{3}$ Chiraz Chaalala, MD, ${ }^{4}$ David Roberge, MD, ${ }^{5}$ Alain Weill, MD, ${ }^{3}$ \\ Christian Stapf, MD, ${ }^{6}$ Daniel Roy, MD, ${ }^{3}$ Michel W. Bojanowski, MD, ${ }^{4}$ Tim E. Darsaut, MD, ${ }^{7}$ \\ Ruby Klink, PhD, ${ }^{8}$ and Jean Raymond, MD $^{3}$
}

\begin{abstract}
${ }^{1}$ Service de Neurochirurgie, CHU Cavale Blanche, INSERM UMR 1101 LaTIM, Brest; ${ }^{2}$ Service de Radiologie, CHU Cavale Blanche, INSERM EA 3878 GETBO, Brest, France; '3Department of Radiology, Service of Neuroradiology, Notre-Dame Hospital, Centre Hospitalier de l'Université de Montréal (CHUM), Montreal; ${ }^{4}$ Department of Surgery, Service of Neurosurgery, ${ }^{5}$ Department of Radio-oncology, and ' ${ }^{6}$ epartment of Neurosciences, Centre Hospitalier de I'Université de Montréal (CHUM), Montreal, Quebec; ' ${ }^{2}$ epartment of Surgery, Division of Neurosurgery, University of Alberta Hospital, Mackenzie Health Sciences Centre, Edmonton, Alberta; and ${ }^{~ I n t e r v e n t i o n a l ~ N e u r o r a d i o l o g y ~ L a b o r a t o r y, ~ C e n t r e ~ H o s p i t a l i e r ~ d e ~ l ' U n i v e r s i t e ́ ~ d e ~ M o n t r e ́ a l ~(C H U M) ~}$ Research Centre, Montreal, Quebec, Canada
\end{abstract}

OBJECTIVE The management of brain arteriovenous malformations (bAVMs) remains controversial. The Treatment of Brain AVMs Study (TOBAS) was designed to manage patients with bAVMs within a clinical research framework. The objective of this study was to study trial feasibility, recruitment rates, patient allocation to the various management groups, and compliance with treatment allocation.

METHODS TOBAS combines two randomized care trials (RCTs) and a registry. Designed to be all-inclusive, the study offers randomized allocation of interventional versus conservative management to patients eligible for both options (first $\mathrm{RCT}$ ), a second RCT testing the role of preembolization as an adjunct to surgery or radiotherapy, and a registry of patients managed using clinical judgment alone. The primary outcome of the first RCT is death from any cause or disabling stroke (modified Rankin Scale score > 2) at 10 years. A pilot phase was initiated at one center to test study feasibility, record the number and characteristics of patients enrolled in the RCTs, and estimate the frequency of crossovers.

RESULTS All patients discussed at the multidisciplinary bAVM committee between June 2014 and June 2016 ( $n=107$ ) were recruited into the study; 46 in the randomized trials ( 23 in the first RCT with 21 unruptured bAVMs, 40 in the second RCT with 17 unruptured bAVMs, and 17 in both RCTs), and 61 patients in the registry. Three patients crossed over from surgery to observation (first RCT).

CONCLUSIONS Clinical research was successfully integrated with normal practice using TOBAS. Recruitment rates in a single center are encouraging. Whether the trial will provide meaningful results depends on the recruitment of a sufficient number of participating centers.

Clinical trial registration no.: NCT02098252 (clinicaltrials.gov)

https://thejns.org/doi/abs/10.3171/2017.2.JNS162751

KEY WORDS brain arteriovenous malformation; randomized trial; care trial; ruptured AVM; unruptured AVM; vascular disorders

ABBREVIATIONS AVM = arteriovenous malformation; $b A V M=$ brain AVM; CHUM = Centre Hospitalier de l'Université de Montréal; $m$ RS = modified Rankin Scale; RCT = randomized care trial; TOBAS $=$ Treatment of Brain AVMs Study.

SUBMITTED November 10, 2016. ACCEPTED February 15, 2017.

INCLUDE WHEN CITING Published online September 1, 2017; DOI: 10.3171/2017.2.JNS162751. 
$\mathrm{B}$ RAIN arteriovenous malformations (bAVMs) are uncommon and complex lesions that can rupture and cause morbidity. ${ }^{3,5,17}$ Their management is controversial and varies across institutions and treatment teams ${ }^{6,7}$ Because ruptured bAVMs have a high annual risk of repeat hemorrhage $(4.5 \%-34 \%),{ }^{23}$ interventional treatment is perhaps advisable $e^{17,24}$ at least in some patients, despite the absence of evidence from randomized controlled trials that benefits outweigh the risks of treatment. ${ }^{2}$ For patients with unruptured bAVMs, hemorrhages occur less frequently $(0.9 \%-8 \% /$ year), and the best management is more controversial. ${ }^{12,15}$

A recent randomized controlled trial showed that medical management of unruptured AVMs was superior to any interventional treatment. ${ }^{14,15,20}$ The study has prompted numerous editorials and critiques, and the clinical community remains unsure of the best way to manage patients with bAVMs. ${ }^{12}$ In addition to the question of whether unruptured bAVMs should be treated, a second question arises when a curative treatment is planned: Is adjunctive embolization in patients with ruptured or unruptured bAVMs beneficial for patients destined to be treated with surgery or radiosurgery? Although embolization by itself can eradicate a bAVM, ${ }^{21}$ and although it may improve the safety and efficacy of surgical or radiosurgical treatments, whether it is worthwhile to accept the additional risks of endovascular treatment for a greater overall benefit to patients is uncertain. ${ }^{16}$

Surgical trials are notoriously difficult to conduct, particularly when they concern widely different but accepted and practiced therapies..$^{10,19}$ In the present case, trials are even more difficult to conduct because bAVMs are rare and heterogeneous lesions that can be managed using a multiplicity of treatment options. Confronted with these difficulties, the Treatment of Brain AVMs Study (TOBAS) was designed. TOBAS differs from conventional trials because it is not simply an experiment designed to answer a research question intended to improve the care of future patients. TOBAS was designed in the spirit of care trials, pragmatic randomized care trials (RCTs) facilitating practice under uncertainty in the best interest of current patients. ${ }^{18}$ Patients are offered promising but yet-to-bevalidated interventions as a $50 \%$ chance of getting the intervention and a 50\% chance of being managed according to the previously validated treatment (observation when none exists)..$^{18}$ Other unusual features of TOBAS include the absence of selection criteria, and an algorithmic decision-making process that, at the time of a multidisciplinary meeting, combines clinical judgment and prerandomization to facilitate the participation of clinicians and patients in the randomized portions of the study. The study is designed to include all patients with bAVMs presenting to participating centers, to offer randomized allocation of curative versus conservative management to patients eligible for both options (first RCT), randomized allocation of adjunctive preembolization when appropriate (second $\mathrm{RCT}$ ), or a registry of patients managed according to clinical judgment alone.

Care trials and prerandomization have been used infrequently in the past. Prior to expansion to international centers, this study sought to assess, in real-world practice, whether TOBAS is capable of accomplishing the stated study purpose: to integrate clinical research and care, and provide a framework within which all current patients with bAVMs can be managed. We report on the proportions and characteristics of patients who were offered randomized allocation versus participation in the multimodal registry, and estimate compliance with the allocated treatments.

\section{Methods \\ Study Design}

TOBAS is a prospective RCT and registry of various management options for patients with bAVMs, including resection, radiation therapy, endovascular embolization, alone or combined, and observation. ${ }^{8,11}$ It is an all-inclusive framework combining clinical research and care, designed in the spirit of care trials. ${ }^{18}$ Patients are offered a management strategy allocated by an algorithmic process combining the clinical judgment of a multidisciplinary team answering 5 simple questions and randomization, when judged appropriate. TOBAS is compliant with the Declaration of Helsinki; the complete protocol is available at www.clinical-care-trials.org. ${ }^{8}$ This study was registered with the ClinicalTrials.gov database (http://clinicaltrials. gov), and its registration number is NCT02098252.

\section{Study Hypotheses}

Two primary hypotheses correspond to the two RCTs nested within TOBAS. The first primary hypothesis is that "interventional management of brain AVM patients eligible for both conservative and curative treatment alternatives will lead to a decrease in the number of poor outcomes (defined as a modified Rankin Scale [mRS] score > 2) from 25 to $15 \%$ at 10 years" (first RCT). At least 540 randomized patients will be necessary to detect the hypothesized reduction of $10 \%$ in poor outcomes. It is too early to estimate the number of crossovers that will occur; analyses using the intention-to-treat principle would require a larger number, according to the proportion of crossovers. In theory, each management strategy should be separately validated, which would mean that for each modality, we would eventually need to recruit 540 patients or more.

The second primary hypothesis is that presurgical or preradiosurgical embolization of bAVMs treatable with or without preembolization can decrease the number of treatment failures (defined as failure to achieve angiographic cure with a good clinical outcome [mRS score > 2]) from $20 \%$ to $10 \%$ (second RCT); 440 patients will be required to detect the hypothesized 10\% increase (from 80\% to 90\%) in the rate of success, defined as complete bAVM eradication without the occurrence of a disabling complication.

\section{Outcomes}

The primary outcome of the first RCT is death from any cause or disabling stroke (hemorrhage or infarction revealed by imaging and resulting in an mRS score $>2$ ) at 10 years. The primary outcome of the second RCT is treatment failure, defined as failure to achieve angiographic cure with a good clinical outcome (mRS score > 2 ) at 1 year. The secondary outcome measures for the two RCTs include assessment of the primary outcome, serious 
TABLE 1. Results for the first 107 patients in the TOBAS algorithmic process, an all-inclusive framework using clinical judgment and two potential randomizations

\begin{tabular}{|c|c|c|c|}
\hline Questions (Q) & Answers & Mode of Allocation & Allocation or Next Algorithmic Step \\
\hline $\begin{array}{l}\text { Q1: Is the patient being considered for } \\
\text { curative treatment? }\end{array}$ & No $(n=36)$, yes $(n=71)$ & $\begin{array}{l}\text { Clinical judgment } \\
\text { alone }\end{array}$ & No: observation registry; yes: go to Q2 \\
\hline $\begin{array}{l}\text { Q2: What is the primary curative method } \\
\text { planned? }\end{array}$ & $\begin{array}{l}\text { Surgery }(n=49) \text {, radiotherapy } \\
\quad(n=15) \text {, embolization }(n=7)\end{array}$ & $\begin{array}{l}\text { Clinical judgment } \\
\text { alone }\end{array}$ & Go to $Q 3$ \\
\hline $\begin{array}{l}\text { Q3: Is conservative management a rea- } \\
\text { sonable alternative option to curative } \\
\text { treatment? }\end{array}$ & No $(n=48)$, yes $(n=23)$ & $\begin{array}{l}\text { Clinical judgment } \\
\quad \& \text { randomization }\end{array}$ & $\begin{array}{l}\text { No: treatment registry if curative method = emboliza- } \\
\text { tion; no: go to Q4 if curative method = surgery or } \\
\text { radiotherapy; yes: RCT } 1 \text { then go to Q4 }\end{array}$ \\
\hline $\begin{array}{l}\text { Q4: Is preembolization being considered } \\
\text { prior to surgery or radiotherapy? }\end{array}$ & No $(n=19)$, yes $(n=45)$ & $\begin{array}{l}\text { Clinical judgment } \\
\text { alone }\end{array}$ & No: treatment registry; yes: go to $\mathrm{Q} 5$ \\
\hline $\begin{array}{l}\text { Q5: Can surgery or radiation therapy be } \\
\text { offered w/o preembolization? }\end{array}$ & No $(n=36)$, yes $(n=40)$ & $\begin{array}{l}\text { Clinical judgment } \\
\quad \& \text { randomization }\end{array}$ & $\begin{array}{l}\text { No: keep same allocation (treatment registry or RCT } \\
\text { 1); yes: keep same allocation if RCT } 1 \text { = conserva- } \\
\text { tive management; yes: RCT } 2 \text { in remaining cases }\end{array}$ \\
\hline
\end{tabular}

adverse events, ruptures, symptomatic strokes, and angiographic occlusion of the lesion at 1 and 5 years.

\section{Treatment Allocation and Randomization}

All patients with bAVMs examined at participating centers are eligible for TOBAS. The sole selection criterion is the presence of a bAVM. Allocation of management choices is a combination of clinical judgment by the multidisciplinary team, and two potential randomization procedures according to a simple algorithmic process composed of 5 questions on the online case report form (Table 1).

Patients best managed conservatively according to clinical judgment alone are offered participation in the observation registry. When interventional management is considered, the primary mode of intervention (embolization, radiosurgery, or surgery) is selected according to clinical judgment prior to randomized allocation in the first RCT comparing conservative and interventional management when appropriate. When embolization is used as a primary mode of intervention, as is sometimes performed in some centers for some patients, it is selected (according to clinical judgment) as the answer to the second question of the algorithmic process ("What is the primary curative method planned? Surgery, radiation therapy, or endovascular treatment"). These patients will be analyzed in a stratified fashion, within the first RCT, or within the treatment registry, depending on the response to the third question ("Is conservative management a reasonable alternative option to curative treatment? Yes or No"). When embolization is used only as an adjunct prior to surgery or radiation therapy, either surgery or radiation is selected as the primary mode of intervention, and the patient is then a potential candidate for the second RCT. Eligible patients, according to clinical judgment, can be included in the second RCT comparing surgery or radiation therapy with or without preembolization.

Patients considered ineligible for conservative management, and for the second RCT, are offered participation in the dedicated treatment registry (embolization alone, surgery alone, radiosurgery alone, surgery with preembolization, and radiosurgery with preembolization). Patients eligible for one or both RCTs are prerandomized, but all patients are informed of the treatment alternatives and of which portion of their treatment plan has been randomly allocated.

Randomization is stratified according to intended primary interventional management group (surgery, radiation therapy, or embolization). A minimization algorithm balances groups with respect to clinical presentation (hemorrhagic versus all other presentations) and to Spetzler-Martin AVM grade (I or II vs III-V). ${ }^{22}$

\section{Pilot Phase}

The pilot phase recruited patients in a single center (Centre Hospitalier de l'Université de Montréal [CHUM]) between June 2014 and June 2016. The protocol is under ethics board review at other sites. We report characteristics of patients and bAVMs according to age, clinical presentation, ruptured or unruptured status, and Spetzler-Martin grading. We analyzed the proportion of patients recruited in each randomized group and in the multimodal registry, the differences between groups, and compliance with treatment allocation.

\section{Results}

Between June 2014 and June 2016, all patients discussed by the multidisciplinary committee on the management of patients with bAVMs at the CHUM $(n=107)$ were enrolled. Sixty-one patients were entered into the registry and 46 into the RCTs.

Twenty-three patients were included in the first RCT (conservative vs interventional management; Fig. 1, Table 2). Most $(21 / 23,91 \%)$ were patients with unruptured bAVMs. One patient with a hemorrhage 12 years prior was neurologically intact at the time of randomization; another patient presented following a subarachnoid hemorrhage from a ruptured prenidal aneurysm, which had been embolized. The primary curative method planned for these 23 patients was surgery in 17 , embolization in 2, and radiosurgery in 4 patients.

Of the 40 patients considered for the second RCT (preembolization or not), 6 were allocated to conserva- 


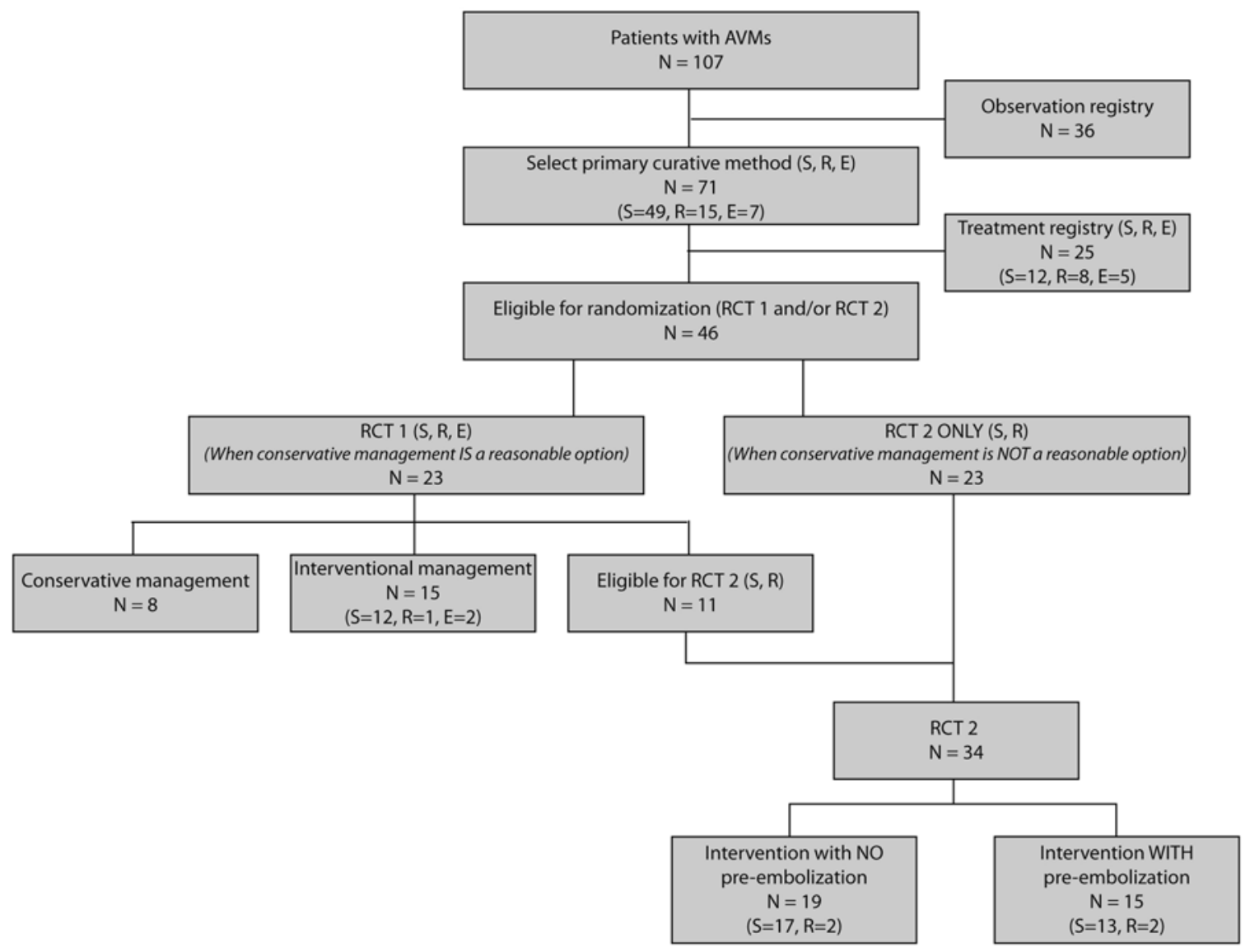

FIG. 1. TOBAS trial profile. $E$ = embolization; $R$ = radiotherapy; $S=$ surgery.

tive management according to the first randomized trial, leaving 34 patients for prerandomization (including $11 \mathrm{pa}-$ tients participating in both RCTs; Tables 2 and 3, Fig. 1); $23(67 \%)$ of 34 presented with a ruptured bAVM (43\% of all patients with ruptured bAVMs), most often low grade (26/34 [77\%] Grade I or II). The primary curative method planned was surgery in 35 patients and radiosurgery in 5 .

Thirty-six patients for whom intervention was not considered were enrolled in the observation registry (Table 2, Fig. 1). Patients in this group were older than patients in the other groups (mean age 52 vs 43 years). Most (29/36, $81 \%$ ) had unruptured bAVMs; 29 (55\%) of 53 patients with unruptured bAVMs were included in the observation registry. Intervention was not considered because of a high Spetzler-Martin grade (Grade IV in 6 cases and Grade V in 3); major comorbidities (schizophrenia in 2 cases, mental deficiency in 2 cases, and major disability related to moyamoya disease in 1 patient); patient age ( $>60$ years in 11 patients); refusal of any treatment ( 2 patients); and in 2 patients, Grade II and III bAVMs were judged to be in eloquent locations that did not allow complete eradication without a major deficit. Seven patients with a ruptured bAVM (13\% of all 54 patients with ruptured bAVMs) were observed because of Spetzler-Martin Grade IV or V

TABLE 2. Patients included in TOBAS

\begin{tabular}{lccccc}
\hline \multicolumn{1}{c}{ Variable } & RCT 1 & RCT 2 & Observation Registry & Treatment Registry & Total \\
\hline No. of patients & 23 & 34 & 36 & 25 & 107 \\
\hline Mean age/range (yrs) & $38 / 19-82$ & $35 / 11-64$ & $52 / 15-79$ & $42 / 12-64$ & $43 / 11-82$ \\
\hline Men & 11 & 16 & 16 & 9 & 47 \\
\hline Women & 12 & 18 & 20 & 16 & 60 \\
\hline Unruptured bAVM & 21 & 11 & 29 & 23 & 53 \\
\hline Ruptured bAVM & 2 & 23 & 7 & 16 & 54 \\
\hline S-M Grades I \& II & 14 & 26 & 19 & 9 & 67 \\
\hline S-M Grades III-V & 9 & 8 & 17 & 40 \\
\hline
\end{tabular}

S-M = Spetzler-Martin. ${ }^{22}$ 
TABLE 3. Patients included in the second RCT $(n=40)$

\begin{tabular}{lcccc}
\hline \multicolumn{1}{c}{ Randomization 2 } & Intervention w/o Preembolization & Intervention w/ Preembolization & Conservative Management After RCT 1 & Total \\
\hline No. of patients & 19 & 15 & 40 \\
\hline Mean age/range (yrs) & $37 / 11-58$ & $33 / 11-64$ & $45 / 33-56$ & 6 \\
\hline Unruptured bAVM & 8 & 3 & 0 & $37 / 11-64$ \\
\hline Ruptured bAVM & 11 & 12 & 3 & 17 \\
\hline S-M Grades I \& II & 16 & 10 & 3 & 21 \\
\hline S-M Grades III-V & 3 & 5 & 10 \\
\hline
\end{tabular}

bAVMs in 4 patients, comorbidities in 2 patients (obstructive pulmonary disease and mental deficiency), and a noncompact nidus in a 70-year-old patient.

Intended management of all patients is summarized in Table 4. Of the 71 patients considered for interventional management, the intended primary mode of curative treatment was embolization in 7 , radiosurgery in 15 , and surgery in 49 patients. Twenty-five patients considered ineligible for RCTs were entered into the corresponding treatment registry; 23 patients presented with a ruptured bAVM, and 2 patients with unruptured bAVMs with neurological symptoms were treated within the treatment registry by palliative partial embolization.

In the first RCT $(n=23)$, among the 8 patients allocated to the conservative management arm, 1 patient was treated at 6 months because of progressive hemianopsia due to occipital edema caused by partial venous thrombosis, managed by embolization and surgery. Of the 15 patients allocated to the treatment arm, 7 were treated, 5 are waiting for curative treatment, and 3 patients refused surgery.

\section{Discussion}

The study attempted to assess whether TOBAS could meet the clinical demand for a pragmatic trial framework context within which to manage patients with bAVMs. Results show that virtually all patients with bAVMs can be included in TOBAS. Approximately 50\% (53/107) of patients had unruptured bAVMs, and 40\% (21/53) of those who were eligible for curative or conservative management were prerandomized in the first RCT. The final management choice, which included the randomized allocation, was judged to be optimal care by the treating team in $22(96 \%)$ of 23 patients, but 3 patients (13\%) refused the allocated treatment (surgery) and were managed conservatively. Most patients with ruptured bAVMs $(46 / 54,85 \%)$ were offered treatment, and almost all patients considered for adjunctive preembolization $(23 / 25,92 \%)$ were included in the second RCT comparing curative treatment with or without preembolization. Older patients with unruptured bAVMs or higher-grade lesions were mostly observed, and ruptured or symptomatic bAVMs were mostly treated within the registry.

TOBAS was able to guide a multidisciplinary team of clinicians in the management of patients with a complex neurovascular condition. Designed in the spirit of care trials, TOBAS does not involve added visits, risks, or costs beyond routine clinical practice; registration and case report forms are simple and designed to be completed within minutes by clinicians. ${ }^{18}$
The apparent complexity of the study protocol is deceptive. It reflects the necessity of using clinical research vocabulary to clearly expose the underlying scientific method and explain how clinical results will be counted. However, at the time of practice, when a patient with a bAVM is presented at the multidisciplinary meeting and entered online, the algorithmic questions appear only natural to pose and answer; they serve as guidelines to clinical decision making and at the same time reminders that some treatments cannot be acted on in the same way as those that have previously been shown to be beneficial. The process was adaptable to all circumstances, accepted rapidly, and is now adopted by the multidisciplinary team of our institution.

The lack of selection and the prerandomized design ${ }^{25}$ that was chosen in the pilot center may have facilitated recruitment of patients with unruptured bAVMs in the first RCT: 23 patients in 2 years, a definite improvement, as compared with 3 patients recruited in the same center in 6 years for the previous trial that used conventional randomization to compare conservative and curative management. ${ }^{15}$ One potential problem with using prerandomization is the risk of trading poor recruitment for an excessive number of crossovers. ${ }^{9,25}$ Crossover rates $(3 / 23,13 \%)$ are acceptable so far but need to be closely monitored because they complicate intent-to-treat analyses and necessitate an adjustment in the total number of patients to be enrolled to achieve meaningful results. The fact that all crossovers

TABLE 4. Intended management of all patients $(n=107)$

\begin{tabular}{ccc}
\hline Variable & Unruptured & Ruptured \\
\hline S-M Grades I \& II & & \\
\hline No. of patients & 31 & 36 \\
\hline Mean age/SD (yrs) & $50 / 18$ & $40 / 18$ \\
\hline Surgery & 12 & 27 \\
\hline Embolization & 2 & 3 \\
\hline Radiosurgery & 0 & 4 \\
\hline Observation & 17 & 2 \\
\hline S-M Grades III-V & & \\
\hline No. of patients & 22 & 18 \\
\hline Mean age/SD (yrs) & $42 / 16$ & $51 / 16$ \\
\hline Surgery & 5 & 0 \\
\hline Embolization & 2 & 8 \\
\hline Radiosurgery & 3 & 5 \\
\hline Observation & 12 &
\end{tabular}


were from curative to conservative management is reassuring, considering that all treatments have yet to prove their merits. We believe that facilitating recruitment in a care trial is primarily performed for the benefit of current patients, but the scientific goal of the enterprise cannot be used to force patients to get treatments they would normally refuse. ${ }^{18}$ The alternatives have their own difficulties.

The ARUBA trial (A Randomized Trial of Unruptured Brain Arteriovenous Malformations) used conventional randomization and experienced recruitment difficulties; it showed the immediate morbidity of curative attempts at eradicating unruptured bAVMs in highly selected patients, but conclusions remain controversial. ${ }^{12}$ One alternative is to stop all treatments, as some have suggested..$^{13}$ Other authors are requesting a national or international registry to monitor the clinical outcomes of various treatments. ${ }^{1}$ This option may be scientifically too weak to eventually justify risky preventive treatments. In addition, while patients are typically more inclined to participate because decisions are made just like standard care, registries offer no protection against unvalidated interventions. Both options are available in TOBAS, by participating in the corresponding observation or treatment registries. Between these 2 extremes, we believe many patients will be best managed within the context of a randomized trial, by being offered a $50 \%$ chance of having access to a curative treatment and a $50 \%$ chance of escaping the potential morbidity of a treatment that may be revealed to be useless or harmful. The substantial number of patients needed to reach meaningful conclusions will require the collaboration of multiple centers, and the task is daunting. In the meantime, the most important contribution of this novel study design may be that the trial offers a clinical research context for prudently practicing interventions that have yet to be shown beneficial. ${ }^{4}$ Finally, the worst-case scenario from a scientific perspective (poor recruitment in the RCTs, or an excessive number of crossovers and no valuable randomized results) would still result in the collection, within TOBAS, of data from 2 observational studies: an observation and a treatment registry.

By recording all patients managed at each center, the TOBAS registry may provide a portrait of the kinds of patients actually managed in each center. This may help estimate the future generalizability of the RCTs. The allinclusive registry and the RCTs are integrated; thus, the registry may promote the RCTs by regularly reminding clinicians of the existence of the trials. It may also identify clinical concerns (adverse events in alarming frequency, for example). Finally, the registry can encourage a culture of multicenter participation in studies questioning the merits of our interventions.

The pilot center has empirically used embolization as an adjunctive treatment of bAVMs for 30 years. This strategy has been appreciated by surgeons and radiation therapists on our multidisciplinary team, who believe their respective treatments are safer and more effective once partial embolization has been performed. The fact that in doing so we are adding risks up front, but with no proven benefit later, has been the foundation of the second RCT questioning the merits of preembolization. The second RCT was this time more frequently used for ruptured bAVMs. Again, we believe that until benefits are shown, the trial may actually protect half the participants from the risks of an intervention that may prove useless or harmful, as compared with management based on clinical judgment alone, when patients are likely to accept embolization as a "prudent" preliminary step to the intervention they agreed to receive (surgery or radiotherapy).

The present study has many limitations, including a small number of patients, incomplete follow-up periods, and treatments that have yet to be performed. Thus, the number of potential crossovers may have been underestimated. The main limitation of this study is that it was a single-center experience. Whether this experience can be generalized to other practices remains to be seen. Other general limitations of the TOBAS trial have been discussed elsewhere. ${ }^{8,11}$

\section{Conclusions}

The TOBAS trial was well accepted by multidisciplinary clinicians at a single center. The design of the trial appears to have found real-world utility in managing all patients with bAVMs. Recruitment rates into the RCTs have been encouraging, but the number of crossovers will need to be closely monitored. Whether the trial will provide meaningful answers will depend on the recruitment of a sufficient number of participating centers.

\section{Acknowledgments}

Development of the online database and randomization site was made possible through a professorial support grant to Dr. Raymond from the Department of Radiology, Radio-oncology, and Nuclear Medicine at the University of Montreal.

\section{References}

1. Bambakidis NC, Cockroft KM, Hirsch JA, Connolly ES, Amin-Hanjani S, Meyers PM, et al: The case against A Randomized Trial of Unruptured Brain Arteriovenous Malformations: misinterpretation of a flawed study. Stroke 45:2808-2810, 2014

2. Bendok BR, El Tecle NE, El Ahmadieh TY, Koht A, Gallagher TA, Carroll TJ, et al: Advances and innovations in brain arteriovenous malformation surgery. Neurosurgery $\mathbf{7 4}$ (Suppl 1):S60-S73, 2014

3. Berman MF, Sciacca RR, Pile-Spellman J, Stapf C, Connolly ES Jr, Mohr JP, et al: The epidemiology of brain arteriovenous malformations. Neurosurgery 47:389-397, 2000

4. Bojanowski MW, Magro E, Darsaut T, Raymond J: Improving arteriovenous malformation research and care. J Neurosurg 122:1250, 2015 (Letter)

5. Brown RD Jr: Unruptured brain AVMs: to treat or not to treat. Lancet Neurol 7:195-196, 2008

6. Cockroft KM, Chang KE, Lehman EB, Harbaugh RE: AVM Management Equipoise Survey: physician opinions regarding the management of brain arteriovenous malformations. $\mathbf{J}$ Neurointerv Surg 6:748-753, 2014

7. Cockroft KM, Jayaraman MV, Amin-Hanjani S, Derdeyn CP, McDougall CG, Wilson JA: A perfect storm: how A Randomized Trial of Unruptured Brain Arteriovenous Malformations' (ARUBA's) trial design challenges notions of external validity. Stroke 43:1979-1981, 2012

8. Darsaut TE, Magro E, Gentric JC, Batista AL, Chaalala C, Roberge D, et al: Treatment of Brain AVMs (TOBAS): study protocol for a pragmatic randomized controlled trial. Trials 16:497, 2015 
9. Darsaut TE, Raymond J: Barrow Ruptured Aneurysm Trial. J Neurosurg 117:378-380, 2012

10. Ergina PL, Cook JA, Blazeby JM, Boutron I, Clavien PA, Reeves BC, et al: Challenges in evaluating surgical innovation. Lancet 374:1097-1104, 2009

11. Magro E, Gentric JC, Darsaut TE, Batista AL, Chaalala C, Roberge D, et al: [Treatment of brain AVMS (TOBAS): a randomized controlled trial and registry.] Neurochirurgie 62:197-202, 2016 (Fr)

12. Magro E, Gentric JC, Darsaut TE, Ziegler D, Bojanowski MW, Raymond J: Responses to ARUBA: a systematic review and critical analysis for the design of future arteriovenous malformation trials. J Neurosurg 126:486-494, 2017

13. Mohr JP, Hartmann A, Kim H, Pile-Spellman J, Stapf C: Viewpoints on the ARUBA trial. AJNR Am J Neuroradiol 36:615-617, 2015

14. Mohr JP, Kejda-Scharler J, Pile-Spellman J: Diagnosis and treatment of arteriovenous malformations. Curr Neurol Neurosci Rep 13:324, 2013

15. Mohr JP, Parides MK, Stapf C, Moquete E, Moy CS, Overbey JR, et al: Medical management with or without interventional therapy for unruptured brain arteriovenous malformations (ARUBA): a multicentre, non-blinded, randomised trial. Lancet 383:614-621, 2014

16. Morgan MK, Davidson AS, Koustais S, Simons M, Ritson EA: The failure of preoperative ethylene-vinyl alcohol copolymer embolization to improve outcomes in arteriovenous malformation management: case series. J Neurosurg 118:969-977, 2013

17. Ogilvy CS, Stieg PE, Awad I, Brown RD Jr, Kondziolka D, Rosenwasser R, et al: AHA Scientific Statement: Recommendations for the management of intracranial arteriovenous malformations: a statement for healthcare professionals from a special writing group of the Stroke Council, American Stroke Association. Stroke 32:1458-1471, 2001

18. Raymond J, Darsaut TE, Altman DG: Pragmatic trials can be designed as optimal medical care: principles and methods of care trials. J Clin Epidemiol 67:1150-1156, 2014

19. Raymond J, Darsaut TE, Molyneux AJ: A trial on unruptured intracranial aneurysms (the TEAM trial): results, lessons from a failure and the necessity for clinical care trials. Trials 12:64, 2011

20. Ross J, Al-Shahi Salman R: Interventions for treating brain arteriovenous malformations in adults. Cochrane Database Syst Rev (7):CD003436, 2010

21. Saatci I, Geyik S, Yavuz K, Cekirge HS: Endovascular treat- ment of brain arteriovenous malformations with prolonged intranidal Onyx injection technique: long-term results in 350 consecutive patients with completed endovascular treatment course. J Neurosurg 115:78-88, 2011

22. Spetzler RF, Martin NA: A proposed grading system for arteriovenous malformations. J Neurosurg 65:476-483, 1986

23. Stapf C, Mast H, Sciacca RR, Choi JH, Khaw AV, Connolly ES, et al: Predictors of hemorrhage in patients with untreated brain arteriovenous malformation. Neurology 66:1350-1355, 2006

24. Starke RM, Komotar RJ, Hwang BY, Fischer LE, Garrett MC, Otten ML, et al: Treatment guidelines for cerebral arteriovenous malformation microsurgery. Br J Neurosurg 23:376-386, 2009

25. Zelen M: Randomized consent designs for clinical trials: an update. Stat Med 9:645-656, 1990

\section{Disclosures}

Dr. Roberge reports that he has received support of non-studyrelated clinical or research effort from Elekta, Varian Medical Systems, and Siemens Healthineers; and he reports being a consultant to Brainlab AG.

\section{Author Contributions}

Conception and design: Raymond, Magro, Gentric, Roberge, Weill, Stapf, Bojanowski, Darsaut. Acquisition of data: Raymond, Magro, Gentric, Batista, Kotowski, Chaalala, Roberge, Weill, Stapf, Roy, Bojanowski, Darsaut. Analysis and interpretation of data: Raymond, Magro, Gentric, Batista, Kotowski, Chaalala, Roberge, Weill, Stapf, Bojanowski, Darsaut, Klink. Drafting the article: Raymond, Magro, Gentric, Darsaut, Klink. Critically revising the article: all authors. Reviewed submitted version of manuscript: all authors. Approved the final version of the manuscript on behalf of all authors: Raymond. Statistical analysis: Raymond, Magro, Gentric, Klink. Administrative/technical/material support: Klink. Study supervision: Raymond, Magro, Gentric.

\section{Correspondence}

Jean Raymond, CHUM-Notre-Dame Hospital, Interventional Neuroradiology (NRI), 1560 Sherbrooke East, Pavillion Simard, Rm. Z12909, Montreal, QC H2L 4M1, Canada. email: jean. raymond@umontreal.ca. 